\title{
Poloxamer 188 enhances apoptosis in a human leukemia cell line
}

\author{
NOBUO AOKI ${ }^{1}$, MICHIKO TAMURA $^{1}$, JUNKO H. OHYASHIKI ${ }^{2}$, MAKI SUGAYA $^{1}$ and HISASHI HISATOMI ${ }^{1}$ \\ ${ }^{1}$ Department of Materials and Life Science, Seikei University; \\ ${ }^{2}$ Intractable Disease Research Center, Tokyo Medical University, Tokyo, Japan
}

Received February 26, 2010; Accepted May 6, 2010

DOI: $10.3892 / \mathrm{mmr} 00000314$

\begin{abstract}
Poloxamer block copolymers have been studied in multiple applications as drug delivery systems (DDS). These A-B-A amphiphilic block copolymers up-regulate the expression of selected genes in cells and alter genetic responses to antineoplastic agents in cancer. One example is poloxamer 188, also known as pluronic F68, which may be promising as a carrier in DDS. To clarify the possible mechanistic role of pluronic F68 in several leukemia cell lines, we examined whether pluronic F68-inducible factors were capable of causing apoptosis. The influence of pluronic F68 on the cell lines was examined using a comprehensive analysis. It was found that treatment of K562 cells with 6\% pluronic F68 resulted in $\mathrm{G}_{2} / \mathrm{M}$ phase arrest of the cell cycle, followed by caspase activation and the accumulation of apoptotic cells. When used as a carrier in a DDS, pluronic F68 may provide a synergistic effect on the drug of interest. Although the mechanisms behind the function of pluronic F68 are not fully understood, the results suggests that pluronic F68 may act as a useful carrier in DDS for the purpose of leukemia therapy.
\end{abstract}

\section{Introduction}

The management of leukemia has become complex due to the availability of improved diagnostic procedures and lifeprolonging or even curative treatment strategies. However, many technical difficulties (e.g., the prevention of adverse drug reactions) have to be overcome before effective leukemia therapy can be realized. One of the ways to enhance drug uptake and bioavailability is through the application of efficient drug

Correspondence to: Dr Hisashi Hisatomi, Laboratory of Cellular and Molecular Biochemistry, Department of Materials and Life Science, Seikei Universty, 3-3-1 Kichijoji Kita-machi, Musashino-shi, Tokyo 180-8633, Japan

E-mail: hisatomi@st.seikei.ac.jp

Abbreviations: DDS, drug delivery systems; CML, chronic myelogenous leukemia; PEG, polyethylene glycol; PBMC, peripheral blood mononuclear cells; PI, propidium iodide

Key words: poloxamer, pluronic F68, drug derivery system, apoptosis delivery systems (DDS) $(1,2)$. Although the concept of DDS is not new, significant progress has recently been made in the treatment of a variety of diseases (3-5). A recent therapeutic strategy in anti-cancer treatment is the application of DDS, such as micelles, liposomes and copolymers (6-8). To convey a sufficient dose of drug to the lesion, suitable carriers of drugs are also needed. Poloxamer (also known as pluronic ${ }^{\circledR}$ ) surfactants are synthetic copolymers of ethylene oxides and propylene oxide. Pluronics are biocompatible and have been widely used for wound healing, drug and gene delivery and tissue engineering (9-11). Pluronic block copolymers have been recognized as pharmaceutical excipients by the US and British Pharmacopoeia. Poloxamer 188 (also known as pluronic ${ }^{\circledR}$ F68) is the most commonly used copolymer for drug delivery in clinical trials $(12,13)$. Pluronic F68, a copolymer of poly(oxyethlene)-poly(oxypropylene) -poly(oxyethylene), has been used extensively in a variety of pharmaceutical formulations, including the delivery of low molecular mass drugs and polypeptides (14).

To understand how pluronic F68 acts in vitro, we cultured the human chronic myelogenous leukemia (CML) cell line K562 with pluronic F68 or polyethylene glycol (PEG). PEG (MW 7000-9000) is comparable to pluronic F68 (MW 8350) in terms of its molecular weight. Using flow cytometry, pluronic F68 was determined to be a specific inhibitor of cell proliferation in K562 cells. It is possible that it may be effective as a DDS for the treatment of leukemia via the synergistic effect of pluronic F68, as the carrier, and the drug.

\section{Materials and methods}

Cell culture. All solvents and reagents, except when otherwise specified, were purchased from Sigma-Aldrich (MO, USA) at the highest purity grade. Pluronic F68, a copolymer of poly(oxyethylene)-poly(oxypropylene)-poly(oxyethylene) with a POE/POP ratio of 20/80, was obtained from Wako Junyaku (Kyoto, Japan). PEG (molecular weight 7,000-9,000 kDa; mean $8,000 \mathrm{kDa}$ ) was obtained from Wako Junyaku. The K562, HL60, NALM-6 and Molt-4 cell lines (Table I) were grown at $37^{\circ} \mathrm{C}$ in a humidified incubator with $5 \% \mathrm{CO}_{2} / 95 \%$ air in RPMI 1640 medium supplemented with $10 \%$ fetal bovine serum (Gibco, NY, USA), $1 \mathrm{mM}$ of sodium pyruvate, $200 \mathrm{IU} / \mathrm{ml}$ of penicillin and $200 \mu \mathrm{g} / \mathrm{ml}$ of streptomycin. After subculture for $48 \mathrm{~h}$, the cells were treated with $6 \%$ pluronic F68 or PEG for $48 \mathrm{~h}$. Peripheral blood mononuclear cells (PBMC) derived from healthy volunteers were isolated by 
Table I. Cell lines used in the study.

\begin{tabular}{llll}
\hline Name & Tissue & Morphology & \multicolumn{1}{c}{ Genetics } \\
\hline K562 & Peripheral blood & Lymphocyte-like & Chronic myelogenenous leukemia \\
HL60 & Peripheral blood & Lymphocyte-like & Premyeloblastic cell \\
NALM-6 & Peripheral blood & Lymphocyte-like & B cell leukemia \\
Molt-4 & Peripheral blood & Lymphocyte-like & T cell leukemia \\
\hline
\end{tabular}
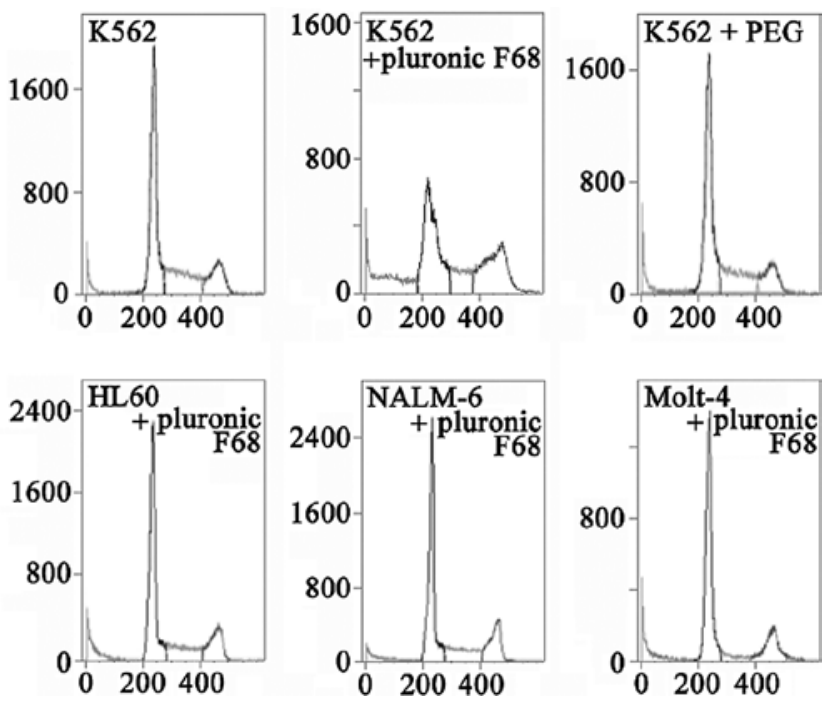

Figure 1. DNA histograms of cell lines in the absence or presence of $6 \%$ pluronic F68 or 6\% PEG. The K562 cell line with pluronic F68 exhibited a detectable apoptosis signal (sub- $\mathrm{G}_{1}$ ) and was synchronized in the $\mathrm{G}_{2} / \mathrm{M}$ phase of the cell cycle. Other cell lines incubated with $6 \%$ pluronic F68 experienced no changes in either the apoptosis signal (sub- $\mathrm{G}_{1}$ ) or the state of the cell cycle $\left(\mathrm{G}_{0} / \mathrm{G}_{1}, \mathrm{~S}\right.$ and $\left.\mathrm{G}_{2} / \mathrm{M}\right)$.
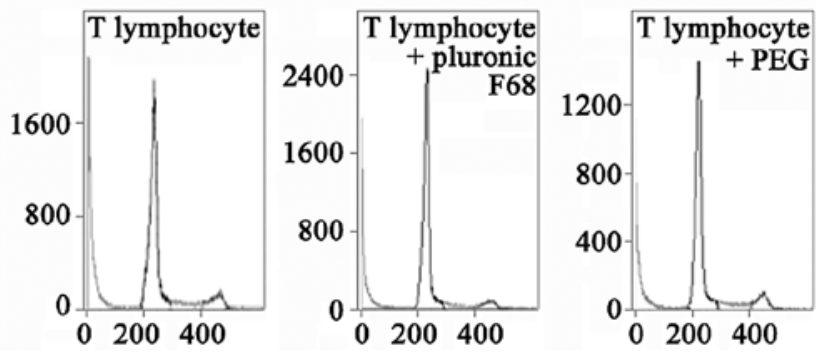

Figure 2. DNA histograms of T lymphocytes derived from healthy volunteers. T lymphocytes incubated with $6 \%$ pluronic F68 or 6\% PEG experienced no changes in the apoptosis signal or the state of the cell cycle.

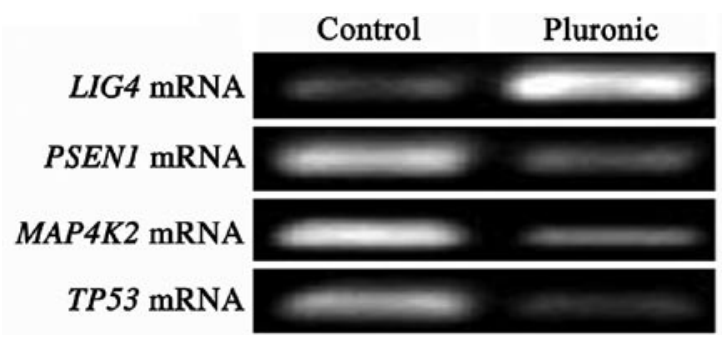

Figure 3. Detection of pluronic F68-inducible mRNA in the K562 cell line in the absence or presence of $6 \%$ pluronic F68. Control, the K562 cell line treated without pluronic F68; Pluronic, the K562 cell line treated with 6\% pluronic F68.
Ficoll-based lymphosepar I (Immuno-Biological Laboratories, Gunma, Japan) centrifugation. Monocytes were eliminated from the PBMC and the PBMC were incubated at $37^{\circ} \mathrm{C}$ for $1 \mathrm{~h}$ on a collagen-coated dish. Any non-adherent cells were recovered. The cells were cultured for $24 \mathrm{~h}$ with $100 \mathrm{ng} / \mathrm{ml}$ of anti-CD3 mbs (Beckman Coulter, CA, USA) and $40 \mu \mathrm{g} /$ $\mathrm{ml}$ of phytohemagglutinin-P (Gibco, CA, USA) in RPMI1640 medium. After subculture for $48 \mathrm{~h}$, the cells were treated with $6 \%$ pluronic F68 or PEG for $48 \mathrm{~h}$.

Cell growth inhibition. Cell cycle distribution was determined by flow cytometry using a Quanta SC (Beckman Coulter, CA, USA). At $48 \mathrm{~h}$ after incubation, apoptosis was detected by Annexin V and propidium iodide (PI) staining. Cell cycle arrest was detected by PI staining.

DNA microarray. mRNA were hybridized on a DNA chip as described previously (15). Total RNA was extracted using QuickGene mini-80 (Fujifilm, Odawara, Japan) according to the manufacturer's instructions. The final RNA preparations were quantified by their absorbances at $260 \mathrm{~nm}$ using a NanoDrop ND-1000 (Biomedical Science, Tokyo, Japan). cDNA was prepared by incubating $22 \mu \mathrm{g}$ of total RNA with cyanine 5- or 3-labeled dUTP (Applied Biosystems, CA, USA) in the presence of an oligo dT primer. cDNA probes were hybridized on a Human a la Carte array (Novus Gene, Tokyo, Japan) with a GeneTAC Hybstation (Genomis solutions, Ann Arbor, MI, USA). After hybridization, DNA chips were scanned using a GenePix 4000B (Axon Instruments, CA, USA). The factor that had been obtained by the DNA chip assay was confirmed by RT-PCR. cDNA was prepared by incubating DNase-treated total RNA $(0.1 \mu \mathrm{g})$ with M-MLV reverse transcriptase (Invitrogen, CA, USA) in the presence of random primers (Invitrogen). The reaction parameters were $95^{\circ} \mathrm{C}$ for $30 \mathrm{sec}, 60^{\circ} \mathrm{C}$ for $40 \mathrm{sec}$, and $72^{\circ} \mathrm{C}$ for $30 \mathrm{sec}$ for 40 cycles, followed by a 5 -min extension at $72^{\circ} \mathrm{C}$ using Paq 5000 DNA polymerase (Stratagene, CA, USA). Half of the PCR product was separated using electrophoresis in TBE buffer on a $2.0 \%$ agarose gel, then stained with ethidium bromide and detected under ultraviolet light.

\section{Results}

Cell growth inhibition. The K562 cell line with pluronic F68 exhibited a detectable apoptosis signal with Annexin V. The other cell lines, HL60, NALM-6 and Molt-4, when incubated with pluronic F68, had undetectable apoptosis signals. In addition, when the $\mathrm{K} 562$ cell line was incubated with pluronic F68, it was synchronized in the $\mathrm{G}_{2} / \mathrm{M}$ phase of the cell cycle 
(Fig. 1). None of the cell lines incubated with PEG exhibited a detectable apoptosis signal or experienced changes in the state of their cell cycle. T lymphocytes derived from healthy volunteers incubated with pluronic F68 or PEG exhibited no changes in the apoptosis signal or the state of the cell cycle (Fig. 2).

DNA microarray. After the mRNA expression was normalized to the GAPDH housekeeping gene expression, we identified pluronic F68-inducible target mRNAs. LIG4 mRNA was up-regulated by $>5$-fold, while PSEN1, MAP4K2 and TP53 mRNA were down-regulated by $>5$-fold. These mRNAs were confirmed to be increased or decreased using both RT-PCR (Fig.3) and the DNA chip assay.

\section{Discussion}

In the present study, induction by pluronic F68 arrested the $\mathrm{G}_{2} / \mathrm{M}$ phase of the cell cycle in the K562 BCR-ABL-mediated CML cell line. Furthermore, the $\mathrm{G}_{2} / \mathrm{M}$ arrest consequentially induced the apoptosis. This cell-specific death was only observed in the K562 cell, and not observed in the other cell lines, HL60, NALM-6 and Molt-4. Moreover, this K562specific apoptosis was induced only by pluronic F68, and not by PEG. PEG did not influence the cell proliferation of any of the cell lines. In addition, the influence of cell proliferation on T lymphocytes with pluronic F68 was not confirmed. Pluronic F68 is not only a K562-specific carrier of a DDS, but also acts as an anti-cancer drug in CML. No previous reports have found a relationship between pluronic F68 and K562 or its function as an apoptotic enhancer; therefore, we have no explanation for the K562-specific $\mathrm{G}_{2} / \mathrm{M}$ arrest observed in the present study. However, the increase or decrease in mRNA clearly shows some features of apoptosis.

Using cDNA microarray analysis, we identified four pluronic F68-inducible target mRNAs, LIG4, PSEN1, $M A P 4 K 2$ and TP53. The up-regulated mRNA was LIG4, while the down-regulated mRNAs were PSEN1, MAP4K2 and TP53. LIG4 is involved in DNA repair and PSEN1, $M A P 4 K 2$ and TP53 are apoptosis-related genes. LIG4, ligase IV forms a complex with the X-ray repair cross complementing protein 4, and further interacts with the DNA-dependent protein kinase (16). Increases in this gene result in apoptosis. PSEN1, presenilins-1, is postulated to regulate amyloid precursor protein processing through its effects on $\gamma$-secretase, an enzyme that cleaves the amyloid precursor protein (17,18). MAP4K2, mitogen-activated protein kinase 2 , is a member of the serine/threonine protein kinase family. This kinase can be activated by TNF- $\alpha$, and has been shown to specifically activate mitogen-activated protein kinases $(19,20)$. The TP53 gene encodes tumor protein $\mathrm{p} 53$, which responds to diverse cellular stresses to regulate the target genes that induce cell cycle arrest, apoptosis, senescence, DNA repair or changes in metabolism (21,22). Decreases in PSEN1, MAP4K2 and TP53 mRNA result in apoptosis.

Many anticancer drugs (e.g., paclitaxel, docetaxel) are generally $\mathrm{G}_{2} / \mathrm{M}$-phase-specific and show an antiproliferative effect on actively dividing cancer cells. These drugs also affect proteins or nucleic acids that are present in normal as well as malignant tissues. Targeted therapy $(23,24)$ using pluronic F68 as a carrier may be more effective than current treatments and less harmful to normal cells. Pluronic F68 appears to possess unique drug-targeted cancer cell interactions in the K5262 cell line. Therefore, we propose a drug delivery strategy for BCR-ABL(+) CML cells that would target not only the fusion transcript, but also downstream signaling.

The current study demonstrates that treatment of K562 cells with $6 \%$ pluronic F68 results in $\mathrm{G}_{2} / \mathrm{M}$ phase arrest of the cell cycle, followed by caspase activation and the accumulation of apoptotic cells. However, the mechanism behind the involvement of the K562-specific DDS carrier in apoptosis remans unclear. Before being applied in clinical studies, numerous validation studies for DDS using pluronic F68 are required. Further studies are critically important for the identification of new carriers to act in DDS for leukemia therapy.

\section{References}

1. Govender T, Ojewole E, Naidoo P and Mackraj I: Polymeric nanoparticles for enhancing antiretroviral drug therapy. Drug Deliv 15: 493-501, 2008.

2. Kumar MN and Kumar N: Polymeric controlled drug-delivery systems: perspective issues and opportunities. Drug Dev Ind Pharm 27: 1-30, 2001.

3. Kshirsagar SJ, Bhalekar MR and Pawar RP: In vitro drug release and in vivo human X-ray studies of ileo-cecal targeting budesonide fast disintegrating tablet. Drug Dev Ind Pharm 31: 1-8, 2009.

4. Nagahara AH, Merrill DA, Coppola G, et al: Neuroprotective effects of brain-derived neurotrophic factor in rodent and primate models of Alzheimer's disease. Nat Med 15: 331-337, 2009.

5. Whitehead KA, Langer R and Anderson DG: Knocking down barriers: advances in siRNA delivery. Nat Rev Drug Discov 8: 129-138, 2009.

6. Letchford $\mathrm{K}$ and Burt $\mathrm{H}$ : A review of the formation and classification of amphiphilic block copolymer nanoparticulate structures: micelles, nanospheres, nanocapsules and polymersomes. Eur J Pharm Biopharm 65: 259-269, 2007.

7. Okuda T, Kawakami S, Yokoyama M, Yamamoto T, Yamashita F and Hashida M: Block copolymer design for stable encapsulation of N-(4-hydroxyphenyl)retinamide into polymeric micelles in mice. Int J Pharm 357: 318-322, 2008.

8. Ruiz MA, Clares B, Morales ME and Gallardo V: Vesicular lipidic systems, liposomes, PLO, and liposomes-PLO: characterization by electronic transmission microscopy. Drug Dev Ind Pharm 34: 1269-1276, 2008.

9. Gaymalov ZZ, Yang Z, Pisarev VM, Alakhov VY and Kabanov AV: The effect of the nonionic block copolymer pluronic P85 on gene expression in mouse muscle and antigenpresenting cells. Biomaterials 30: 1232-1245, 2009.

10. Kabanov AV, Batrakova EV, Sriadibhatla S, Yang Z, Kelly DL and Alakov VY: Polymer genomics: shifting the gene and drug delivery paradigms. J Control Release 101: 259-271, 2005.

11. Ma WD, Xu H, Nie SF and Pan WS: Temperature-responsive, Pluronic-g-poly(acrylic acid) copolymers in situ gels for ophthalmic drug delivery: rheology, in vitro drug release, and in vivo resident property. Drug Dev Ind Pharm 34: 258-266, 2008.

12. Kabanov AV, Batrakova EV and Alakhov VY: Pluronic block copolymers for overcoming drug resistance in cancer. Adv Drug Deliv Rev 54: 759-779, 2005.

13. Soppimath KS, Aminabhavi TM, Dave AM, Kumbar SG and Rudzinski WE: Stimulus-responsive 'smart' hydrogels as novel drug delivery systems. Drug Dev Ind Pharm 28: 957-974, 2002.

14. Toth K, Wenby RB and Meiselman HJ: Inhibition of polymerinduced red blood cell aggregation by poloxamer 188 . Biorheology 37: 301-312, 2000.

15. Ohyashiki JH, Takaku T, Ojima T, Abe K, Yamamoto K, Zhang Y and Ohyashiki K: Transcriptional profiling of human herpesvirus type $\mathrm{B}(\mathrm{HHV}-6 \mathrm{~B})$ in an adult $\mathrm{T}$ cell leukemia cell line as in vitro model for persistent infection. Biochem Biophys Res Commun 329: 11-17, 2005. 
16. Werbrouck J, De Ruyck K, Duprez F, et al: Acute normal tissue reactions in head-and-neck cancer patients treated with IMRT: influence of dose and association with genetic polymorphisms in DNA DSB repair genes. Int J Radiat Oncol Biol Phys 73: 1187-1195, 2009

17. Sherrington R, Rogaev EI, Liang Y, et al: Cloning of a gene bearing missense mutations in early-onset familial Alzheimer's disease. Nature 375: 754-760, 1995.

18. Thinakaran G, Borchelt DR, Lee MK, et al: Endoproteolysis of presenilin 1 and accumulation of processed derivatives in vivo. Neuron 17: 181-190, 1996.

19. Guru SC, Agarwal SK, Manickam P, et al: A transcript map for the $2.8-\mathrm{Mb}$ region containing the multiple endocrine neoplasia type 1 locus. Genome Res 7: 725-735, 1997.

20. Wissing J, Jansch L, Nimtz M, et al: Proteomics analysis of protein kinases by target class-selective prefractionation and tandem mass spectrometry. Mol Cell Proteomics 6: 537-547, 2007.
21. Harris CC: Structure and function of the p53 tumor suppressor gene: clues for rational cancer therapeutic strategies. J Natl Cancer Inst 88: 1442-1455, 1996.

22. Hartwell LH and Kastan MB: Cell cycle control and cancer. Science 266: 1821-1828, 1994.

23. Dung TH, Kim J, Kim MS, Kim JS and Yoo H: Preparation and biophysical characterization of pluronic F127-dendrimer conjugate as a delivery agent of antisense oligonucleotides. J Nanosci Nanotechnol 8: 5326-5330, 2008.

24. Lemieux P, Vinogradov SV, Gebhart CL, et al: Block and graft copolymers and NanoGel copolymer networks for DNA delivery into cell. J Drug Target 8: 91-105, 2000. 